\title{
Filament destabilization and CME release during a long duration flare
}

\author{
F. Zuccarello ${ }^{1}$, L. Contarino ${ }^{1}$, F. Farnik ${ }^{2}$, M. Karlicky ${ }^{2}$, P. Romano ${ }^{3}$, and I. Ugarte-Urra ${ }^{4}$
}

\author{
1 Dipartimento di Fisica e Astronomia - Sezione Astrofisica, Università di Catania, via S. Sofia 78, 95123 Catania, Italy \\ e-mail: fzu@oact.inaf.it \\ 2 Astronomical Institute of the Academy of Sciences of the Czech Republic, 25165 Ondřejov, Czech Republic \\ 3 INAF - Osservatorio Astrofisico di Catania, via S. Sofia 78, 95123 Catania, Italy \\ ${ }^{4}$ College of Science, George Mason University, 4400 University Drive, Fairfax, VA 22030, USA \\ Received 4 April 2011 / Accepted 24 June 2011
}

\section{ABSTRACT}

\begin{abstract}
Context. During complex and long duration solar flares, several filament destabilizations or eruptions can occur that are often related to coronal mass ejections (CMEs).

Aims. We describe the study of an X3.8 long duration event (LDE) that occurred in NOAA 10720 on 17 January 2005 and was characterized by three filament destabilizations and two CMEs.

Methods. Using multi-wavelength data provided by both ground-based instruments and satellites, in addition to MDI magnetograms, we investigated the morphological and magnetic evolution of the active region before and during the LDE.

Results. Our analysis of $\mathrm{H}_{\alpha}$ and $1600 \AA$ Amages showed that initially a two-ribbon structure developed in the central part of the active region, where a filament was previously observed. At a later time, two bright ribbons (in the most eastern side) and a strong brightness increase (at the western outskirt of the active region) were simultaneously observed. In a subsequent time interval, a new pair of ribbons was observed in the western side of the active region. Moreover, a linear force-free field extrapolation helped identify a null point in the central part of the active region.

Conclusions. The initial filament destabilization that occurred in the central part of NOAA 10720 was probably due to magnetic flux emergence and photospheric shearing motions, which caused a slow tether-cutting process beneath the filament. The rearrangement of the magnetic field configuration, occurring in the same area as the location of the null point, changed the magnetic field connectivity in the active region, triggering two filament eruptions in the eastern and western part of the active region and two halo CMEs, in a kind of domino effect.
\end{abstract}

Key words. Sun: activity - Sun: flares - Sun: filaments, prominences - magnetic fields

\section{Introduction}

Solar flares are very energetic events $\left(\sim 10^{28}-10^{32} \mathrm{erg}\right)$ that involve several atmospheric layers and can produce emission across the whole electromagnetic spectrum, from $\gamma$ rays to radio wavelengths, in addition to particle acceleration, heating, and bulk plasma motions (see, e.g. Priest \& Forbes 2000, 2002; Aschwanden 2004).

Our knowledge of flares has greatly improved thanks to multi-wavelengths high-resolution observations, allowing us to follow the event evolution in different atmospheric layers and different energy bands, providing a more complete picture of the overall process. However, despite this improvement, there are still many open problems in our understanding of some aspects of these very complex phenomena. One of these aspects concerns the characteristics of the magnetic configuration of the active region hosting the flare. In this respect, it seems that the key issue is the occurrence of magnetic reconnection processes in sites characterized by strong magnetic field gradients and the presence of null points, separatrices, and current sheets. Therefore, it is clear that very complex, sheared, and twisted magnetic field configurations, containing sufficient amounts of free energy, can more easily give rise to eruption events.

Owing to the different magnetic topology of the flaring active regions that have been studied so far, many models have been presented that are able to reproduce, at least in part, the phenomena observed. These models are based on different driver mechanisms and different magnetic topologies. For instance, the flux emergence model presented by Heyvaerts et al. (1977) assumes that a current sheet forms between a new emerging magnetic flux bundle and an old magnetic flux system, giving rise to magnetic reconnection. In a model based on converging motions (Priest et al. 1994), a flux rope is initially at a stationary height, then evolves through a series of forcefree field configurations as the two footpoints of the field lines approach each other, and finally, when the footpoint distance reaches a critical value, it suddenly increase its height, forming a vertical current sheet. We also highlight the CSHKP 2D standard model (Carmichael 1964; Sturrock 1966; Hirayama 1974; Kopp \& Pneuman 1976) based on the assumption that the initial driver is a rising filament stretching a current sheet, where the Sweet-Parker or Petscheck reconnection can occur. In the twodimensional quadrupolar flare model (Hirose et al. 2001), the initial configuration consists of two parallel arcades pushed toward each other by a converging flow pattern. The X-point above the middle neutral line supports a filament that becomes unstable owing to the tearing-mode instability, triggering anomalous resistivity and fast reconnection.

It appears however that the most complex and most energetic events require an interpretation based on several physical processes that have not yet been included in a single model. 
Among these, there are those events characterized by several filaments involved in the flaring process.

In this context, we recall that in a previous paper (Zuccarello et al. 2009, hereinafter Paper I), describing an X17.2 flare that occurred in NOAA 10486 on 28 October 2003, the event was interpreted on the basis of a domino effect, i.e., a sequence of destabilizing processes that triggered the flare. In this event, the active region was characterized by a multiple arcade configuration and the X17.2 flare was preceded, by $\sim 2 \mathrm{~h}$, by the partial eruption of a filament. This eruption caused reconnection at some null points located in the low atmosphere and a decrease in magnetic tension in the coronal field lines superimposed on other filaments present in the active region. Owing to these processes, these filaments were destabilized and the X17.2 flare occurred.

Moreover, if we consider CME-associated flares, we should also recall the kink and torus instabilities, occurring once the winding of flux rope field lines have reached a certain threshold (see, e.g. Vršnak 1990 and references therein; Török et al. 2010 and references therein). In this context it is also important to consider the tether-cutting process, during which the magnetic reconnection takes place in the vicinity of the magnetic inversion line, causing changes within the flux rope that result in the imbalance of forces (loss of equilibrium) and catastrophe, with the consequent rise of the no longer restrained flux rope (see, e.g. Cheng et al. 2010a and references therein).

Some of the mechanisms cited above are believed to take place during long duration events, that is during flares, often associated with CMEs, that last for several hours. More precisely, these models contain an initial slow phase of tether-cutting reconnection below the flux rope, which can disconnect the magnetic field lines from the footpoints anchored in the photosphere. If this process causes a field restructuring in the arcade overlying the flux rope, this can start its rise towards the higher layers of the solar atmosphere. It is then possible that the flux rope/CME eruption and the magnetic reconnection below the rising plasma mutually feed each other (see, e.g.; Lin 2004; Vršnak et al. 2004; Temmer et al. 2008). Apart from weakening the restraint of the overlying magnetic field, the slow reconnection can increase the magnetic pressure at the bottom of the flux rope, because of an increase in the poloidal field, and also cause an increase in the curvature hoop force directed outwards. Below the rising flux rope, a continuous reduction in magnetic pressure takes place, causing a plasma inflow towards the current sheet that was formed. At this stage, a faster tether-cutting reconnection may set-in, once the flux rope rise increases and the plasma inflow towards the current sheet is accelerated.

Therefore, to improve our understanding of these longduration events where they are characterized by multiple filament eruption or destabilization, in this paper we analyze an X3.8 flare occurred in NOAA 10720 on 17 January 2005 using multi-wavelength data. The outline of the paper is the following: in Sect. 2, we present the data used for the analysis of the event; in Sects. 3 and 4, the global morphology of NOAA 10720 and the flare evolution are described; in Sect. 5, the magnetic configuration and the transport of magnetic helicity in the active region are discussed; in Sect. 6, we present the discussion and in Sect. 7 the conclusions.

\section{Data description}

To study the X3.8 flare that occurred in NOAA 10270, we used Ondřejov radio data $(0.8-2.0 \mathrm{GHz}$ and $3 \mathrm{GHz})$, chromospheric and photospheric images acquired at INAF-Catania Astrophysical Observatory (OACT) respectively in the $\mathrm{H}_{\alpha}$ line center (6562.8 $\AA$ ) and in its red wing (6563.8 ̊), $1600 \AA$ images acquired by TRACE, and hard X-ray data acquired by RHESSI in the $12-100 \mathrm{keV}$ band. Full-disk line-of-sight magnetograms provided by the Michelson Doppler Imager (MDI; Scherrer et al. 1995) were also used to study the magnetic evolution of the active region, while full-disk $195 \AA$ images provided by the Extreme ultraviolet Imaging Telescope (EIT; Delaboudinière et al. 1995) were used as reference images to investigate the coronal configuration of NOAA 10720. Both of the MDI and EIT instruments are onboard the SOlar and Heliospheric Observatory (SOHO; Domingo et al. 1995).

Ondřejov radio data (Jiřička et al. 1993), acquired by the $0.8-2.0 \mathrm{GHz}$ radiospectrograph, have a time resolution of $0.1 \mathrm{~s}$ and cover the time interval 8:38-11:30 UT, while the $3 \mathrm{GHz}$ radio data acquired by the radiometer have a time resolution of 0.01 s and cover the time interval 9:01-11:01 UT.

OACT full-disk images were recorded by a $1200 \times 1360$ CCD detector with a spatial resolution of 2.2 arcsec and a dynamic range of 16 bits. In standard patrol mode, the $\mathrm{H}_{\alpha}$ images in the line center and its red wing with a passband of $0.25 \AA$ are acquired, using the same telescope, with a time cadence of $1 \mathrm{~h}$ and 15 min, respectively, while the $\mathrm{H}_{\alpha}$ images taken on 17 January between 7:51 UT and 12:45 UT have an average time cadence of about $40 \mathrm{~s}$ (time cadence during flares and erupting filaments being adopted), for a total of 291 images.

The $1600 \AA$ TRACE images were taken between 07:05:12 UT and 12:23:24 UT, with a gap during the time interval 09:20-09:55 UT. A total of 2065 images were acquired in the $512 \times 512$ pixel mode, while 294 images were taken in full resolution mode, i.e. $768 \times 768$ pixel, with a pixel size of 0.5 arcsec and a field of view (FoV) of $6.4 \times 6.4$ arcmin; the average time cadence was $\sim 30 \mathrm{~s}$.

The Ramathy High Energy Solar Spectroscopic Imager (RHESSI) spacecraft provides imaging spectroscopy at high temporal and angular resolution in the energy range 3-300 keV (Lin et al. 2002). The RHESSI data used in this paper cover the time intervals 07:20-08:04 UT and 09:40-09:50 UT on 17 January. We reconstructed RHESSI images in $12-25,25-50$, and 50-100 keV energy channels using the CLEAN algorithm (Hurford et al. 2002), which gives a spatial resolution of $\sim 12$ arcsec, and we overplotted contour levels of 50, 60, 70, 80, 90 and $95 \%$ of the peak intensity over $\mathrm{OACT}_{\alpha}$ images.

The MDI magnetograms that we used, characterized by a spatial resolution of $\sim 4$ arcsec, were acquired between 00:00 UT of 12 January and 22:23 UT of 17 January, with a time cadence of $96 \mathrm{~min}$.

The co-alignment of OACT images with the images acquired by the other instruments was done by comparing the OACT images in the $\mathrm{H}_{\alpha}$ red wing (in the following indicated as OACT photospheric images) with the WL images acquired by $\mathrm{SOHO} / \mathrm{MDI}$ that were closer in time. From the inspection of MDI WL contours of the main sunspots present in NOAA 10720 over the OACT photospheric images, we deduced the $x-y$ shifts to apply to the OACT data (for both photospheric and $\mathrm{H}_{\alpha}$ images). In the TRACE $1600 \AA$ data, we deduced the shifts from the overplot of OACT $\mathrm{H}_{\alpha}$ ribbon contours over the TRACE images acquired at the same time.

\section{NOAA 10720 global morphology}

NOAA 10720 was firstly detected at the east limb of the Sun on 10 January 2005, with an average latitude of nine degrees north and a simple $\beta$ magnetic configuration. It disappeared at the west 

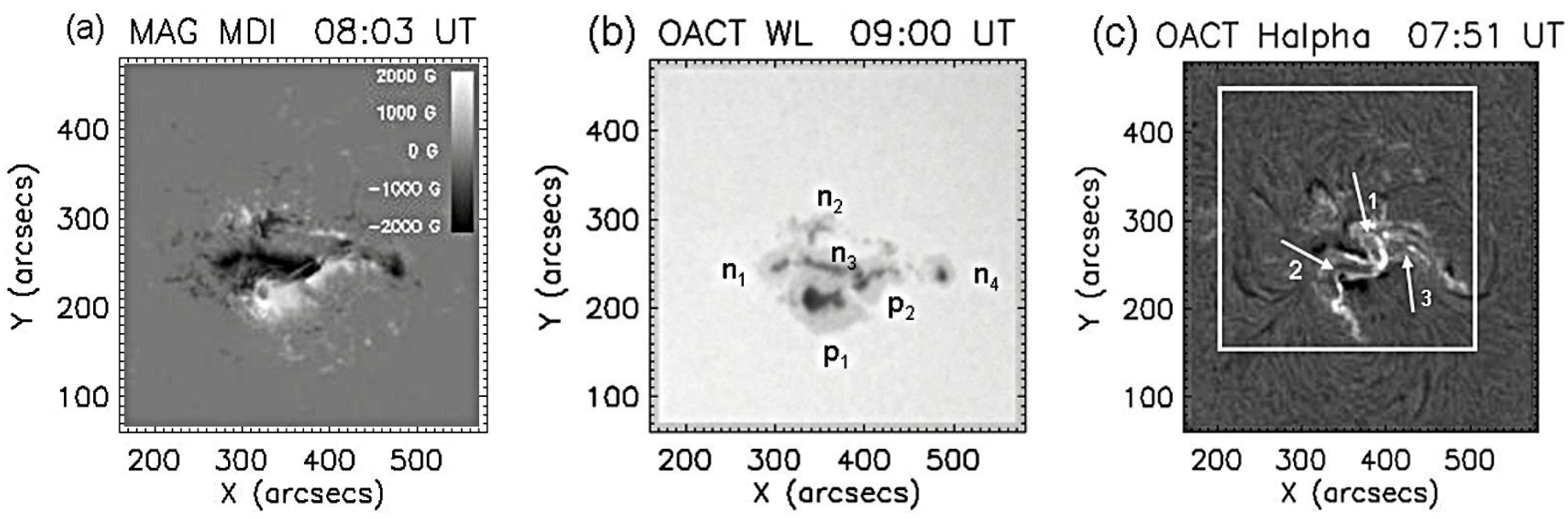

Fig. 1. a) MDI/SOHO magnetogram showing the photospheric magnetic configuration of NOAA 10720 on 17 January 2005 at 08:03 UT (the FoV is $420 \times 420$ arcsec, white (black) indicate positive (negative) polarity); b) photospheric image acquired at OACT on the same day at 09:00 UT, showing the photospheric configuration of the active region; the letters indicate the polarities of the main sunspots: $n_{1}, n_{2}, n_{3}$, and $n_{4}$ indicate the sunspots with negative polarity, while $p_{1}$ and $p_{2}$ indicate the main sunspots with positive polarity; c) OACT $\mathrm{H}_{\alpha}$ image of the chromosphere acquired at 07:51 UT; the white arrows indicate the location of three filaments involved in the flaring process, while the white box indicates the field of view of the images shown in Fig. 3. In these and the subsequent images, north is at the top, west on the right.

limb on 23 January as a complex and large sunspot group. This active region was classified as a super active region (see, e.g.; Romano \& Zuccarello 2008; Criscuoli et al. 2009) because during its passage on the solar disk it displayed a particularly intense flare activity (during the period 14-21 January, five X-class and eighteen M-class flares were observed), and has been extensively studied by other authors (Veronig et al. 2006; Maričić et al. 2007; Temmer et al. 2007; Liu et al. 2008; Park et al. 2008; Temmer et al. 2008, 2009; Wang et al. 2009; Zhao et al. 2009; Cheng et al. 2011). In this paper, we analyze the sequence of events relevant to an X3.8 flare that occurred on 17 January 2005 (peak time 09:52 UT), when the active region was located in the north-west quadrant (N13W29).

The configuration of NOAA 10720 on 17 January is shown in Fig. 1: the MDI magnetogram (Fig. 1a) indicates that the magnetic field shows a prevalently north-south orientation. The magnetic configuration of the active region on this day is $\beta \delta$. In the northern part of the active region, there are four main sunspots (indicated in Fig. 1 b by $n_{1}, n_{2}, n_{3}$, and $n_{4}$ ), characterized by negative polarity; in the southern part, we can see two sunspots, $p_{1}$ and $p_{2}$, with positive polarity.

In the $\mathrm{H}_{\alpha}$ image acquired before the flare peak and reported in Fig. 1c, we can see, in addition to the main sunspots, some filaments inside and around the active region. In particular, the filament indicated by the arrow 1 in the central part of the image is located between sunspots $n_{3}$ and $p_{2}$, filament 2 is located between sunspots $p_{1}$ and $n_{3}$, while filament 3 is located between sunspots $p_{2}$ and $n_{4}$. In Fig. $1 \mathrm{c}$, it is possible to distinguish some bright features, running parallel to the above-mentioned filaments.

We stress that the three filaments might be parts of a single, highly sheared, flux rope. However, the $\mathrm{H}_{\alpha}$ images we analyzed do not allow us to confirm this hypothesis. Therefore, in the following, we refer to these features as separated filaments.

\section{The X3.8 flare evolution}

The GOES-12 X-ray flux reported in Fig. 2a displays a major peak at around 09:52 UT of 17 January, in both the 0.5-4.0 $\AA$ band (red line) and the 1.0-8.0 $\AA$ band (blue line). This peak was associated with an X3.8 flare that occurred in NOAA 10720 , characterized by an extremely long rise phase (from 06:59, when the rise phase starts until 09:52 UT at the peak of the flare) and a quite long main or gradual phase (i.e., the phase occurring from after the peak of the flare until when the soft X-ray brightness returns to the pre-flare values), during which the chromospheric and coronal layers showed brightness variations for more than three hours (long duration event, LDE).

Moreover, if we consider Fig. 2a, we can see that the rise phase presents three abrupt increases, at approximately 06:59, 07:59, and 09:00 UT, respectively, while the very hard X-ray peak starts at 09:42 UT (see Sect. 4.3). In the following, we refer to the time interval 06:59-09:52 UT as the rise phase of the flare and the time interval 09:53-13:00 UT as the main phase of the flare.

From the inspection of Fig. 2a, we can also see that before the X3.8 flare peak, there are an additional two peaks, around 03:10 UT and 06:10 UT, respectively. They correspond to an M2.6 and a C3.9 flare that occurred in NOAA 10720, as deduced from the corresponding NOAA report $\left(\mathrm{see}^{1}\right)$.

The X3.8 flare is correlated with two impulsive halo CMEs (see the LASCO catalog $\mathrm{at}^{2}$ ) observed by the LASCO instrument (Brueckner et al. 1995) on board SOHO. The first CME was initially observed by LASCO C2 at 09:30:05 UT (the probable time of beginning provided by the LASCO catalogue is 09:06 UT), and was characterized by a velocity $v \sim 2094 \mathrm{~km} \mathrm{~s}^{-1}$ and an acceleration $a \sim-119 \mathrm{~m} \mathrm{~s}^{-2}$, while the second CME, which was first observed by LASCO C2 at 09:54:05 UT (the probable time of beginning provided by the LASCO catalogue is 09:42 UT), had a velocity $v \sim 2547 \mathrm{~km} \mathrm{~s}^{-1}$ and an acceleration $a \sim-159 \mathrm{~m} \mathrm{~s}^{-2}$.

\subsection{Results from $\mathrm{H}_{\alpha}$ data}

$\mathrm{H}_{\alpha}$ data acquired at OACT on 17 January are available from 07:51 UT to 12:45 UT (with two gaps: one during the time interval 09:50-09:58 UT, the other between 10:07 and 10:25 UT). These data were reduced following a standard procedure that

\footnotetext{
1 http://www.swpc.noaa.gov/

2 http://cdaw.gsfc.nasa.gov/CME_list
} 


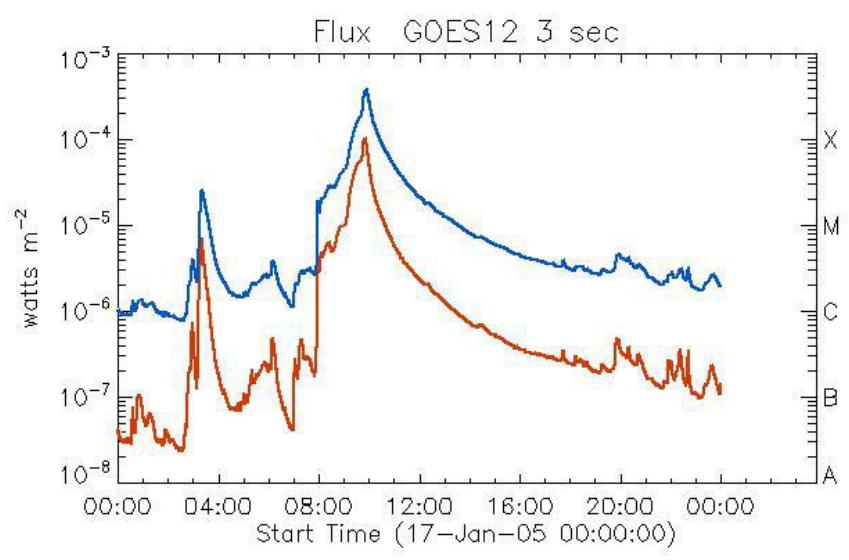

(a)

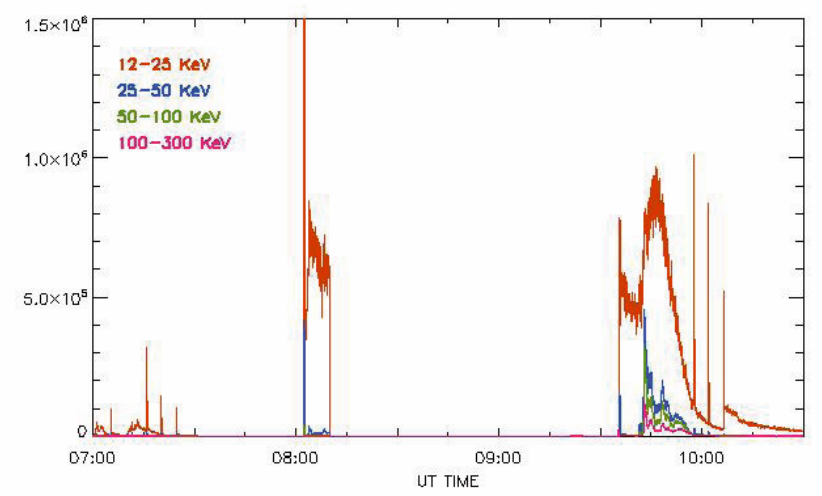

(b)

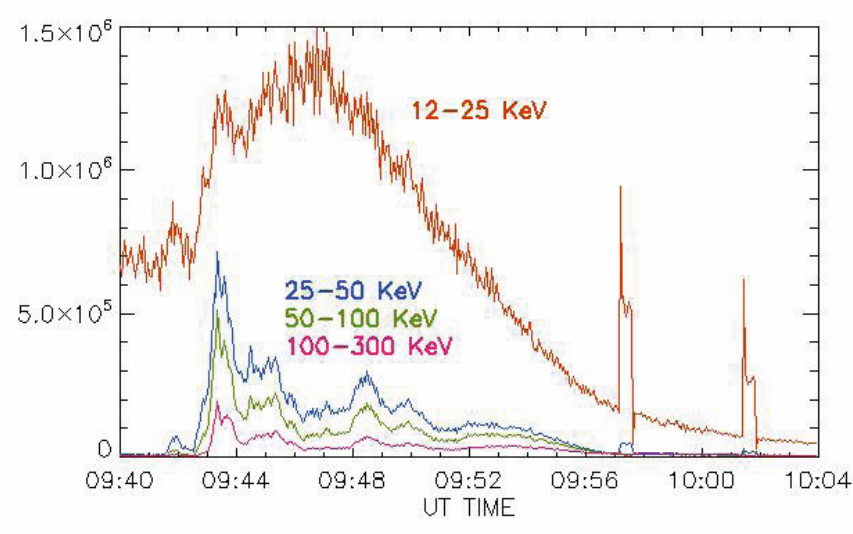

(c)

Fig. 2. a) GOES-12 X-ray flux in the $0.5-4.0 \AA$ band (red line) and in the 1.0-8.0 $\AA$ band (blue line) on 17 January 2005. The graph shows the occurrence of an X3.8 flare, with a peak of intensity at 09:52 UT. b) Light curve showing the X-ray emission measured by RHESSI at different energy bands during the time interval 07:00-10:30 UT. c) RHESSI X-ray light curve showing in more detail the time interval 09:40-10:05 UT. The spikes are probably of instrumental origin.

corrects the images for dark current, gain, flat-field, and darklimb effects. Moreover, a standard procedure for limb definition was used.

In Fig. 3, the first and second rows show the evolution of the flare in the $\mathrm{H}_{\alpha}$ line center. For the sake of clarity, we stress that we use the term destabilization to refer to a filament, when it is subject to a loss of equilibrium that does not necessarily lead to its eruption and expulsion from the solar atmosphere; from an observational point of view, this means that it might show brightenings along its borders, or changes in its length and/or shape, but no evidence of plasma motion. On the other hand, we use the term eruption when the filament rises through the solar atmosphere until it is ejected: from an observational point of view, this means that the images show the plasma, which initially formed the filament, moving from its initial position, and that later images do not show the filament anymore.

The image acquired at 08:01 UT (Fig. 3a) shows that at this time bright ribbons are more evident in the central part of the active region (between 380 and 420 arcsec from the disc center in the $x$ direction) close to the location of filament 1, indicating that filament 1 is being destabilized by some process. At 08:31 UT (Fig. 3b), the previous emission decreased and some brightening was observed on the western side of the AR (at 500 arcsec in the $x$ direction). The shape of the ribbons in the central part appears different in the image acquired at 09:06 UT (Fig. 3c), where we can see that they are more shifted to the east, and their brightness has increased. This behavior seems to indicate that, after the destabilization of filament 1, filament 2 is also subject to a destabilization. At 09:31 UT (Fig. 3d), the ribbons appear brighter in the eastern-central region (between 350 and 400 arcsec in the $x$ direction) close to the initial location of filament 2, while the brightening in the western side has decreased. If we compare the evolution of the ribbons bordering filament 1 (i.e., very limited brightness, in both time and space) with that of filament 2 (more extended, in both time and space), we can presume that the second filament erupted at around or before 09:06 UT, when these ribbons were initially observed, while filament 1 , despite an initial destabilization, was unable to erupt. At 10:00 UT (Fig. 3e), the ribbons increase in brightness again, but this time the majority of the emission comes from a region to the west, between 450 and 550 arcsec, close to filament 3. In the $\mathrm{H}_{\alpha}$ images acquired between 09:45 and 10:00 UT, besides the increase in the emission in these ribbons, it is also possible to clearly distinguish the motion of the plasma within filament 3, which eventually erupted. At 11:00 UT (Fig. 3f), the last two pairs of ribbons are still visible, but their brightness has strongly decreased.

In Fig. 4a, the $\mathrm{H}_{\alpha}$ isocontours relevant to the time 08:03 UT are overplotted on the MDI magnetogram closest in time. At this time we can see that the $\mathrm{H}_{\alpha}$ ribbons are concentrated in the central part of the active region. More precisely, the southern ribbon outlines the eastern part of the magnetic inversion line, while the northern part of the ribbon partially overlaps the inversion line in the proximity of a null point (see Sect. 5) (compare Fig. 1a with Fig. 8). In Fig. $4 \mathrm{~d}$, the $\mathrm{H}_{\alpha}$ isocontours obtained from the image acquired at 11:11 UT (i.e., during the flare main phase), overplotted on the corresponding MDI magnetogram show that at this time the enhanced brightness areas are more distant from the magnetic inversion line.

\subsection{Results from TRACE data}

In Figs. 3g-k, we report a selection of $1600 \AA$ images acquired between 08:19 and 09:11 UT, showing the ribbons before the flare peak. We can clearly see the ribbons in the eastern part of the active region, where filaments 1 and 2 are located, but also a brightness increase concentrated on the western side $(x \sim 450$, $y \sim 270$ ). During the time interval 09:20-10:10 UT, the area between $x=380-450$ and $y=280-340$ seems to be characterized by moving plasma, probably associated with the destabilization and later eruption of filament 3. In Figs. 3j-1, the $1600 \AA$ TRACE images acquired during the flare main phase are shown. 

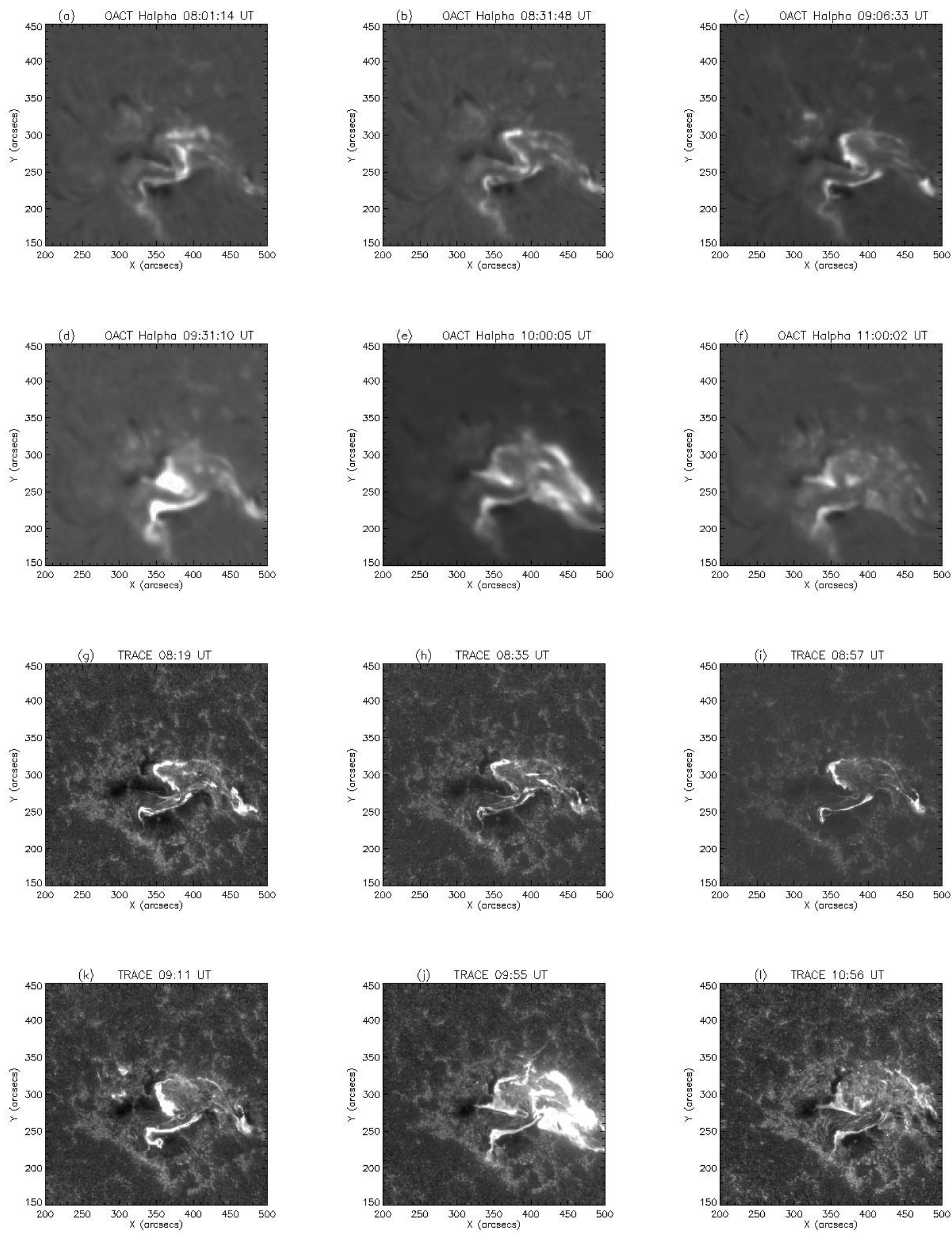

Fig. 3. First and second row: sequence of $\mathrm{H}_{\alpha}$ images acquired at OACT, showing the evolution of the X3.8 flare observed in NOAA 10720. Third and fourth row: sequence of TRACE images, showing the flare ribbon evolution at $1600 \AA$. 

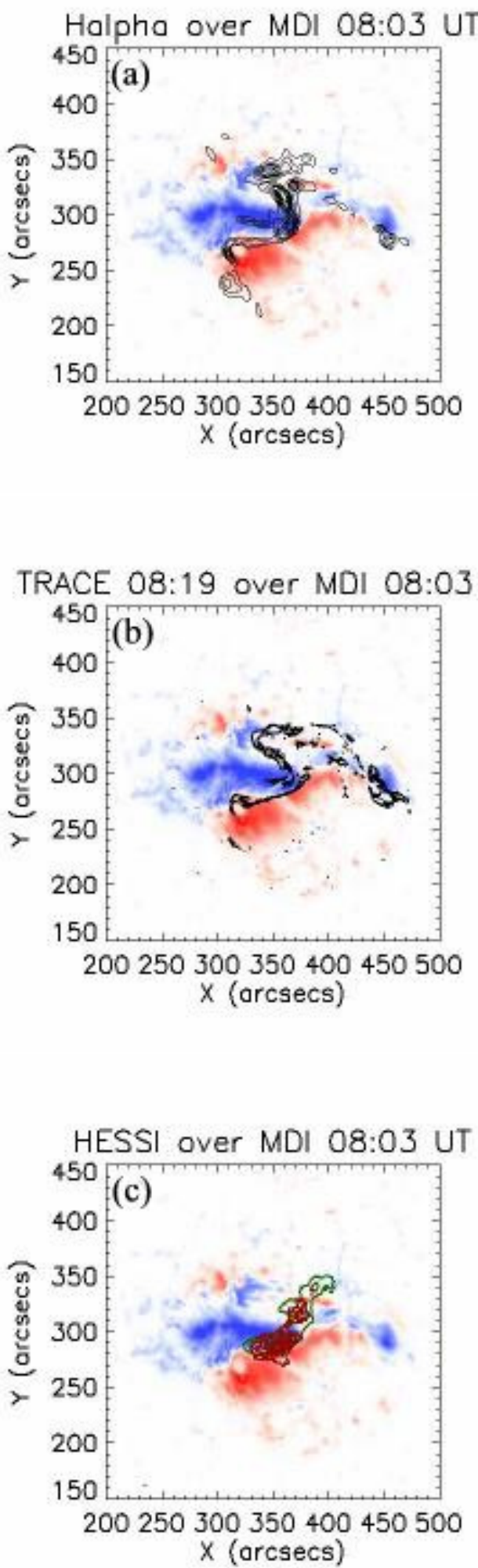
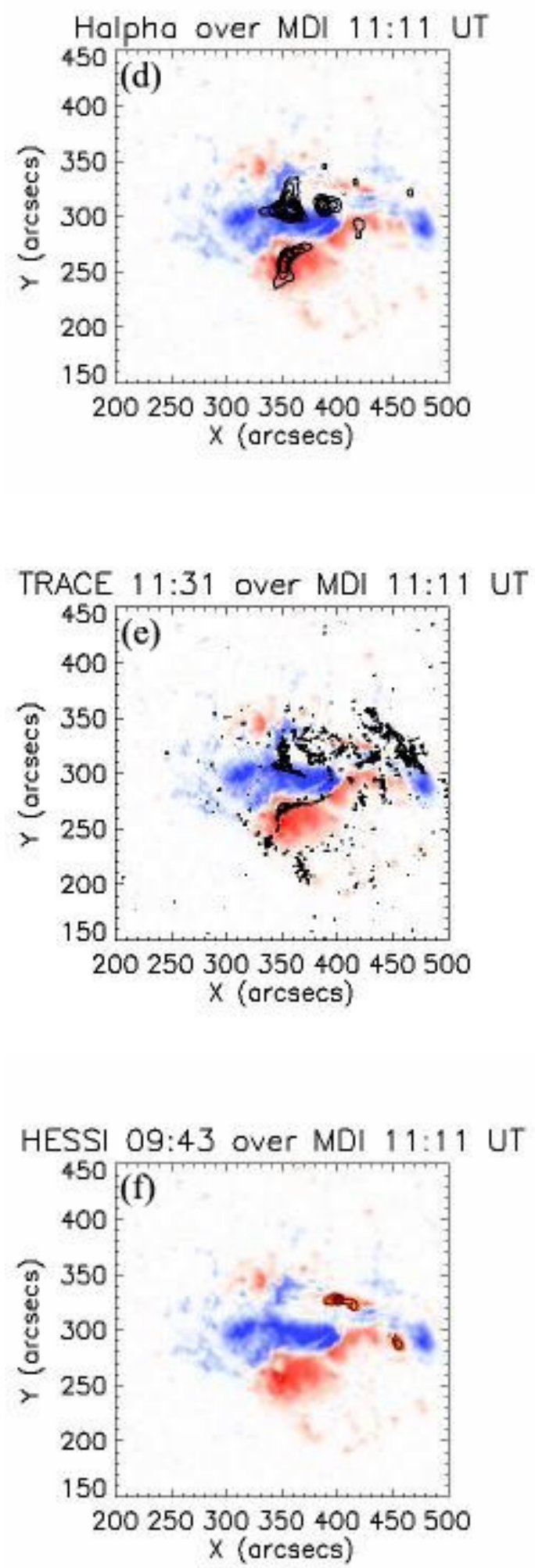

Fig. 4. $\mathrm{H}_{\alpha}$ isocontours overplotted on MDI magnetograms during the rise a) and the main phase d) of the flare; red (blue) colors indicate positive (negative) polarity. Overplots of $1600 \AA$ TRACE isocontours on the corresponding MDI magnetograms during the rise b) and main phase e) of the flare. The contours levels are at 60, 70, 80, and 90\% of maximum $1600 \AA$ intensity in b), while they are at 70, 80, and 90\% in e). Overplots of RHESSI isocontours over MDI magnetograms taken during the rise $\mathbf{c}$ ) and main phase f) of the X3.8 flare. RHESSI data are integrated for $30 \mathrm{~s}$ around 08:04 UT c) and 09:43 UT f). Green isocontours indicate the emission at 12-25 keV, while orange and red isocontours indicate the emission at 25-50 keV and 50-100 keV, respectively. The contour levels are at 50,60, 70, 80, 90, and 95\% of maximum intensity. 


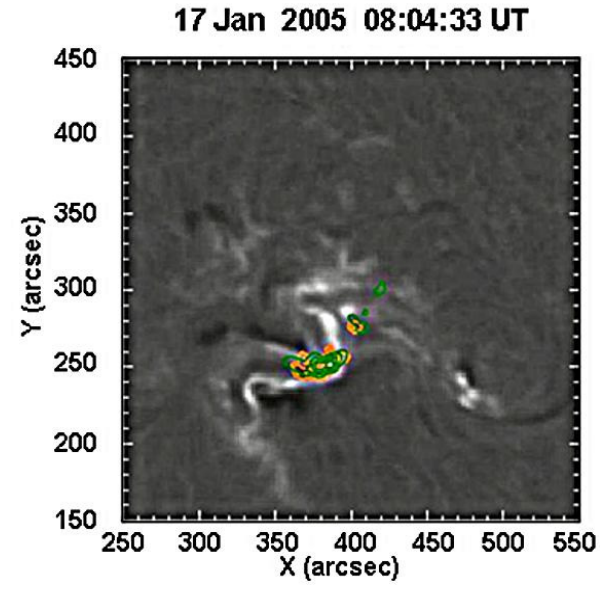

(a)

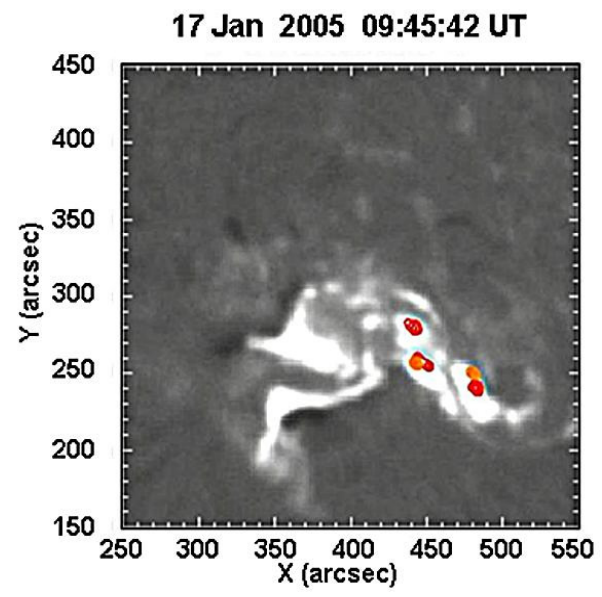

(d)

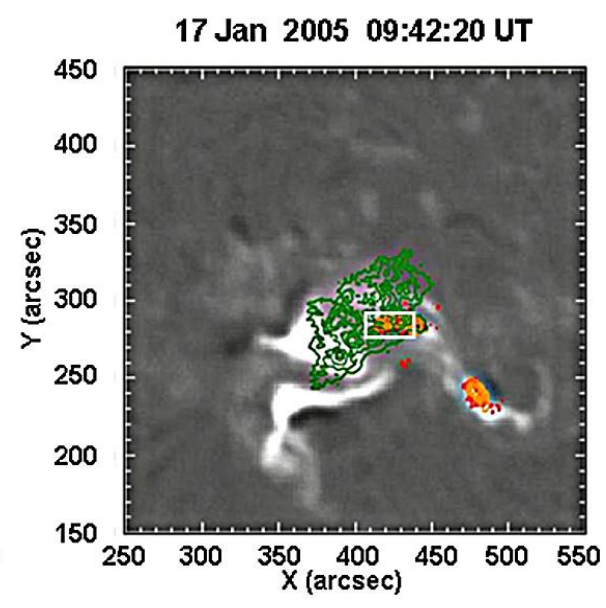

(b)

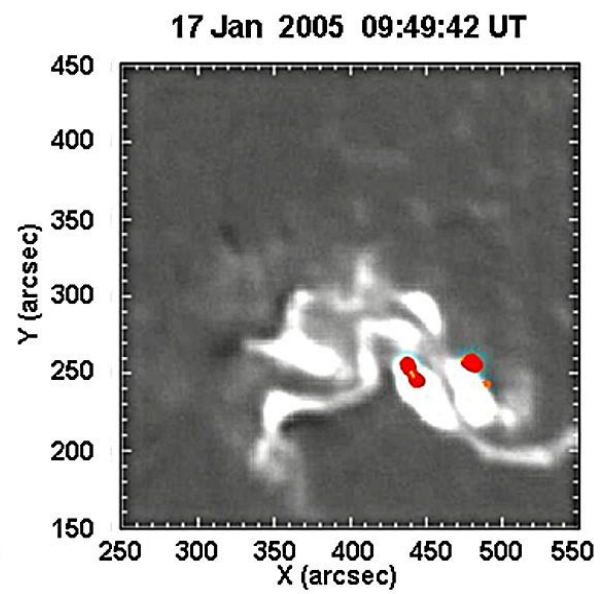

(e)

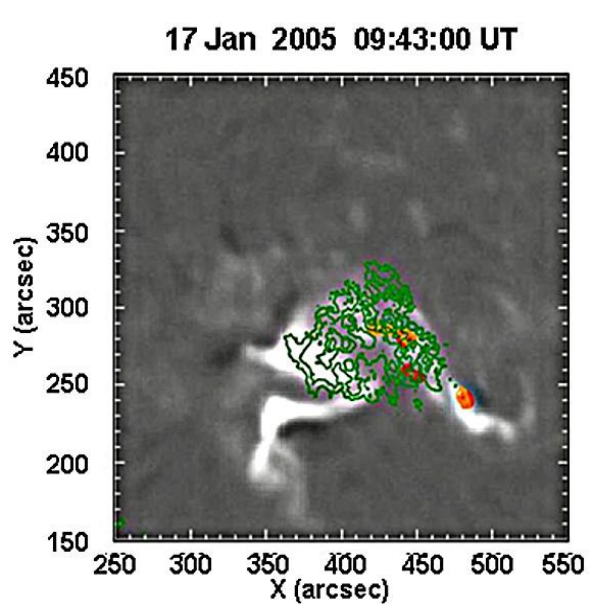

(c)

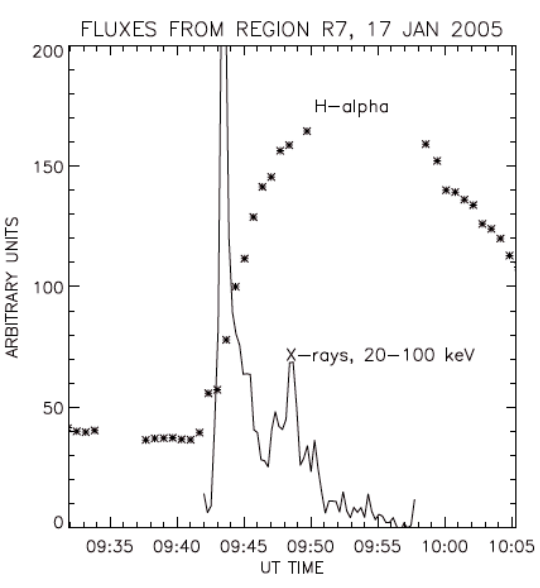

(f)

Fig. 5. a)-e) Overplots of RHESSI isocontours over the $\mathrm{H}_{\alpha}$ images taken at different times during the rise phase of the X3.8 flare. RHESSI data are integrated for $30 \mathrm{~s}$ around 08:04 UT a), 09:42 UT b), 09:43 UT c), 09:45 UT d), and 09:49 UT e). Green isocontours indicate the emission at $12-25 \mathrm{keV}$, while orange and red isocontours indicate the emission at $25-50 \mathrm{keV}$ and $50-100 \mathrm{keV}$, respectively. The contour levels are at 50, 60, $70,80,90$, and $95 \%$ of maximum intensity. f) Light curve showing the $\mathrm{H}_{\alpha}$ and X-ray emission during the time interval 09:32-10:05 UT for the region indicated by the white rectangle in b) (note that there is a gap in the $\mathrm{H}_{\alpha}$ data between 09:50 and 09:58 UT).

These images show that the $1600 \AA$ ribbons have an evolution very similar to those observed in $\mathrm{H}_{\alpha}$.

Figures $4 \mathrm{~b}$ and e present the superimposition of the $1600 \AA$ isocontours on MDI magnetograms during the rise and the main phase, respectively. While in Fig. 4b, the bright $1600 \AA$ ribbons are quite compact and seem to follow the magnetic inversion line and the null point location (see Sect. 5), in Fig. 4e the brightenings are far more spread out.

\subsection{Results from RHESSI data}

In Figs. 2b, c, we report the RHESSI light curves showing the $\mathrm{X}$-ray emission in different energy bands and for different time intervals. In the survey plot reported in Fig. 2b, we can see that the flare evolution is only partially covered by RHESSI (during the time intervals, 07:00-07:30 UT, 08:03-08:10 UT, and 09:40-10:05 UT).

The RHESSI light curve reported in Fig. 2c shows in more detail the X-ray emission during the time interval 09:40-10:05 UT. We can see that the peaks of emission in these energy bands occur from 10 to 6 min before the GOES peak.

In Fig. 5, we report the overplots of RHESSI isocontours over the $\mathrm{H}_{\alpha}$ images acquired at successive times during the rise phase of the X3.8 flare. The green isocontours indicate the emission at $12-25 \mathrm{keV}$, while orange and red isocontours indicate the emission at $25-50 \mathrm{keV}$ and $50-100 \mathrm{keV}$, respectively. The contour levels are at 50,60, 70, 80, 90, and $95 \%$ of maximum intensity.

At 8:04 UT, the RHESSI isocontours overlap with the $\mathrm{H}_{\alpha}$ bright ribbons in the eastern-central part of the active region, close to filaments 1 and 2, while they are more extended in the successive phases (09:42 - and 09:43 UT) and later confined to the western ribbons (09:45-09:49 UT) outlining the borders of filament 3.

In Figs. $4 \mathrm{c}$ and $\mathrm{f}$, we overplot the RHESSI isocontours on the MDI magnetograms at two different times during the rise phase of the flare. At 08:03 UT, the RHESSI isocontours are localized along the central region crossing the main magnetic inversion line (Fig. 4c), while at 09:43 UT, they are smaller and localized on the western side of the active region, apparently above or very close to both the null point (see Sect. 5) and the neutral line (compare Fig. 4d with Fig. 1a).

To study the possible correlation between hard X-ray and $\mathrm{H}_{\alpha}$ emission, we considered the most intense hard X-rays sources. Using 60 images in the time interval 09:42-09:58 UT, we integrated all pixels in the region indicated by a white 
Table 1. Fine structures observed in the $0.8-2.0 \mathrm{GHz}$ frequency range.

\begin{tabular}{lcc}
\hline \hline Time interval (UT) & Frequency $(\mathrm{GHz})$ & Type of fine structure \\
\hline $8: 41: 20-8: 42: 30$ & $0.8-2.0$ & dm type III bursts \\
8:47:30-8:49:30 & $0.9-1.4$ & reverse type III bursts \\
8:50:00-8:54:00 & $0.8-2.0$ & broadband pulsations \\
8:54:00-8:55:00 & $0.8-2.0$ & drifting pulsations \\
8:56:40-8:57:15 & $0.8-1.1$ & DPS \\
8:57:30-8:58:10 & $1.1-2.0$ & narrowband dm-spikes \\
8:58:30-9:02:00 & $0.8-1.6$ & dm-continuum \\
9:02:00-9:03:30 & $0.8-2.0$ & several DPSs \\
9:03:40-9:03:55 & $0.8-2.0$ & bi-directional dm type III bursts \\
& & (starting frequency 1.3 GHz) \\
9:03:40-9:07:00 & $0.8-2.0$ & several DPSs and reverse DPS \\
9:07:00-9:13:00 & $1.0-1.6$ & narrowband pulsations \\
9:13:00-9:15:00 & $0.8-1.3$ & lace bursts \\
9:15:00-9:36:00 & $0.8-2.0$ & broadband pulsations \\
9:35:00-9:40:00 & $0.8-1.0$ & fiber bursts \\
9:40:00-9:42:00 & $0.8-1.4$ & dm-continuum \\
9:43:30-9:55:00 & $0.8-2.0$ & dm-continuum \\
9:55:00-10:49:00 & $0.8-2.0$ & broadband pulsations \\
9:55:00-10:49:00 & $0.8-2.0$ & dm-continuum \\
\hline
\end{tabular}

Notes. DPS means drifting pulsating structure.

rectangle in Fig. 5b and plotted the X-ray light curve (see Fig. 5f, continuous line). In the same figure, we report the $\mathrm{H}_{\alpha}$ light curve for the same area (crosses). We can see that the HXR light curve shows two peaks, with decreasing intensities, at 09:43 and 09:49 UT, respectively, while the $\mathrm{H}_{\alpha}$ emission shows a rise to the maximum starting at the same time as the first HXR peak, and reaches the highest values in coincidence with the second peak, with a delay of $\sim 6$ min from the first HXR peak. A similar inspection carried out on other examples among the most intense hard X-ray sources showed a similar result, with some differences in the time delay between the $\mathrm{H}_{\alpha}$ and the X-ray sources (i.e. between 1 and $10 \mathrm{~min}$ ).

The $\mathrm{H}_{\alpha}-\mathrm{HXR}$ relationship is already well established (see e.g.; Kitahara \& Kurokawa 1990 and references therein). In particular, if we take into account the conclusions drawn by these authors, we can infer some clues about the type of heating that produced the $\mathrm{H}_{\alpha}$ kernel whose light curve is shown in Fig. 5f. Kitahara \& Kurokawa (1990) showed that the $\mathrm{H}_{\alpha}$ light curve might show a primary impulsive peak followed by a later gradual maximum: the first one is generally coincident with the HXR peak and is likely due to heating by a superthermal electron beam, while the second peak, not associated with the HXR peak, should be relevant to heating caused by a heat conduction front. In our case, the $\mathrm{H}_{\alpha}$ emission starts to rise in coincidence with the first HXR peak and lasts for about 5 min: this is consistent with a scenario where the $\mathrm{H}_{\alpha}$ kernel was initially heated by a superthermal electron beam and later by a conduction front, hence supports the hypothesis of a reconnection process at this stage of the event.

\subsection{Results from radio data}

In Fig. 6a, we report the $3 \mathrm{GHz}$ radio flux recorded by the Ondřejov radiometer during the time interval 09:01-11:01 UT. We can see that the $3 \mathrm{GHz}$ radio flux was unusually strong, more than $8000 \mathrm{SFU}$, and in some intervals was even saturated. In this respect, we note that this saturation was reported by this instrument for only five flares during the last solar cycle. The radio flux at $3 \mathrm{GHz}$ rapidly increases in the time interval 9:01-9:25 UT, and then after its saturation at about 9:25-9:40 UT and several peaks, it exponentially decreases to the pre-event value.

In Fig. 6b, the $0.8-2.0 \mathrm{GHz}$ radio spectrum observed at Ondřejov during the time interval 08:38-09:38 UT is shown. The event was very rich in terms of various fine structures: Table 1 summarizes the fine structures recorded in the $0.8-2.0 \mathrm{GHz}$ frequency range.

The detailed analysis showed that at about 8:41 UT the dm type III radio bursts corresponding to electron beams at plasma densities in the interval $7.9 \times 10^{9}-4.9 \times 10^{10} \mathrm{~cm}^{-3}$ (assuming that the emission is at plasma frequency). A series of the reverse drift type III bursts then followed, which indicate that the electron beams propagate downwards, i.e. from the corona towards the denser chromosphere.

An additional phase of the flare is characterized by the first drifting pulsating structure (DPS) at 8:56:40-8:57:15 UT and the narrowband dm-spikes (Fig. 6c), which are signatures of magnetic field reconnection (Kliem et al. 2000; Karlický 1984; Karlický et al. 1996; Bárta et al. 2001). In the following time interval, from 9:02 UT until 9:15 UT, the recorded fine structures show the most dynamical phase of the flare (see Fig. 6d). We observed a series of drifting pulsating structures (i.e. indicating that a series of plasmoids were formed during magnetic field reconnection; for more details, see Karlický 2004). One DPS had a positive frequency drift that is interpreted as the radio emission from the plasmoid moving downwards.

Furthermore, we observed the bi-directional type III burst, which started at $1.3 \mathrm{GHz}$ frequency (i.e. the electron density in the acceleration region can be estimated as $2.0 \times 10^{10} \mathrm{~cm}^{-3}$ ). Then very rare lace bursts were observed at 9:13-9:15 UT (for the interpretation of these bursts, see Karlický et al. 2001).

All these bursts indicate a fast magnetic field reconnection that agrees with the exponential increase in the radio flux at $3 \mathrm{GHz}$ (Fig. 6a). The radio emission is then characterized by strong continua and broadband pulsations until the end of this radio flare at about 11:00 UT. The only exception is the interval 9:35-9:40 UT where the fiber bursts were registered. These bursts are generated by the whistler wave packets propagating along the flare loops upwards into the corona and can be used to determine the flare loop parameters (Rausche et al. 2007). 

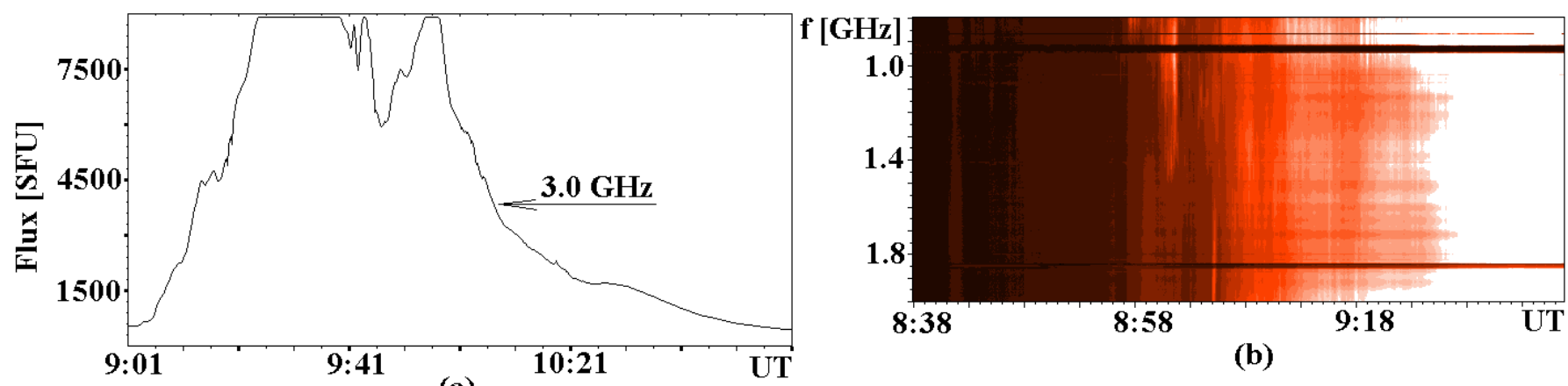

(a)

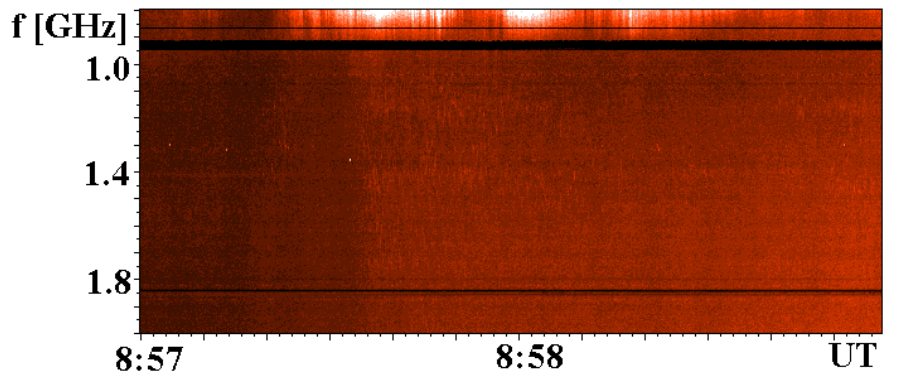

(c)

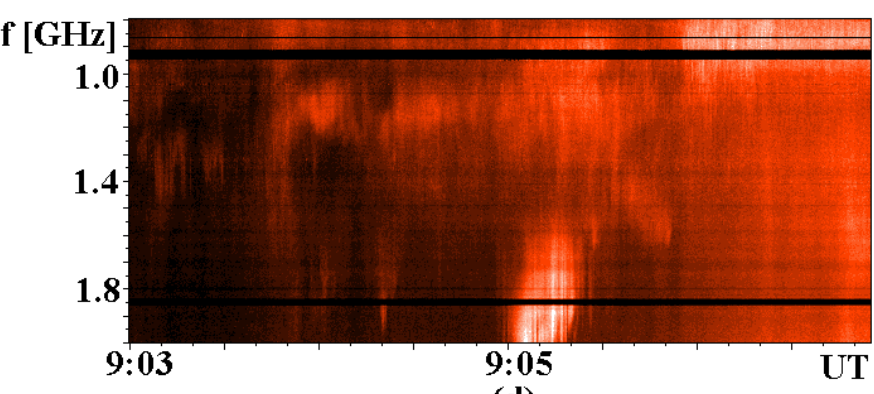

(d)

Fig. 6. a) The $3 \mathrm{GHz}$ radio flux recorded by the Ondřejov radiospectrograph at 09:01-11:01 UT. b) The 0.8-2.0 GHz radio spectrum observed at Ondřejov during the time interval 08:38:00-09:38:00 UT. c) The detailed 0.8-2.0 GHz radio spectrum showing the narrow-band dm-spikes (bright points on the spectrum). The horizontal black lines delineate artificial radio sources. d) The detailed 0.8 $-2.0 \mathrm{GHz}$ radio spectrum showing a series of drifting pulsating structures. At 9:03:40-9:03:55 UT, the bi-directional type III bursts starting at the frequency 1.3 GHz can be seen. Furthermore, the reverse drift DPS is in the time interval 9:05:35-9:05:55 UT in the 1.3-1.65 GHz frequency range.

\section{Magnetic field evolution}

To obtain information about the evolution of the photospheric magnetic field in NOAA 10720, we used the data sets of the MDI/SOHO full-disk observation at $6767.8 \AA$, which provides the line-of-sight component of the magnetic field. To reduce problems arising from geometrical projection effects, we selected only magnetograms where the NOAA 10720 was characterized by a heliographic angle smaller than $45^{\circ}$. Therefore we considered magnetograms taken from 00:00 UT on 12 January to 22:23 UT on 17 January. We corrected all the magnetograms for the angle between the magnetic field direction and the observer's line-of-sight. The data were also aligned and re-gridded with a pixel size of 1 arcsec following the steps described by Chae et al. (2004). Figure 7a reports the evolution of the magnetic flux measured in the whole active region over the field of view of Fig. 7b, i.e., $400 \times 200$ arcsec, during the selected time interval. We can see that during the period analyzed the magnetic flux has values between $1 \times 10^{22} \mathrm{Mx}$ and $5 \times 10^{22} \mathrm{Mx}$, that initially there is a predominance of the negative polarity (thin line), and that later the positive flux (thick line) increases to values greater than the negative flux and on Jan 16 the situation is again inverted.

To determine the magnetic helicity evolution, we studied the horizontal motions of the field line footpoints using the differential affine velocity estimator (DAVE) developed by Schuck (2005). For the DAVE application, we used a window size of 20 arcsec and a time interval between two successive frames of 96 min. Figure $7 b$ reports the horizontal velocity map of the field line footpoints, obtained for 17 January, showing how the magnetic footpoints were then moving at the photospheric level. This map shows that there are large velocity values in the region hosting filament 1: these motions can cause an increase in the stress in the magnetic field supporting the filament.
We then computed the magnetic helicity transport rate by evaluating:

$\frac{\mathrm{d} H}{\mathrm{~d} t}=\int U_{\mathrm{DAVE}}(t, r) A_{\mathrm{p}}(t, r) B_{n}(t, r) \mathrm{d} s$,

where $U_{\text {DAVE }}$ is the estimate of the horizontal motions using the DAVE technique, $\boldsymbol{A}_{\mathrm{p}}$ is the vector potential, and $B_{n}$ is the component of the magnetic field normal to the surface. We calculated $\boldsymbol{A}_{\mathrm{p}}$ using the Fourier transform method described by Chae (2001). In Fig. 7c and d we report the rate of magnetic helicity and the magnetic helicity accumulation as functions of time, respectively. The graph shown in Fig. 7c shows that, with the exception of the time interval 12-13 January, when the rate of magnetic helicity was positive, in the following days, even if the magnetic helicity rate is predominantly negative, there are very abrupt changes in the sign of the magnetic helicity, and one of these changes is temporarily correlated with the X3.8 flare (see the dotted vertical line in Fig. 7c).

The graph shown in Fig. 7d indicates that initially the accumulated magnetic helicity of NOAA 10720 is positive, while from Jan. 13 this parameter is increasingly negative. This last result agrees with the helicity hemispheric rule, implying that negative/positive magnetic helicity is associated with northern/southern active regions. The value of the accumulated magnetic helicity just before the X3.8 flare occurrence is $-2.9 \times$ $10^{43} \mathrm{Mx}^{2}$. Interestingly, after the X3.8 flare occurrence, the rate of magnetic helicity changes sign (compare Fig. 7c with Fig. 7d). This behavior agrees with the results obtained by Smyrli et al. (2010), who analyzed the trend of magnetic helicity accumulation in active regions hosting halo CMEs and found that, in the sample analyzed, the CME events associated with X-class flares display an abrupt change in magnetic helicity at the time of the flare occurrence. 


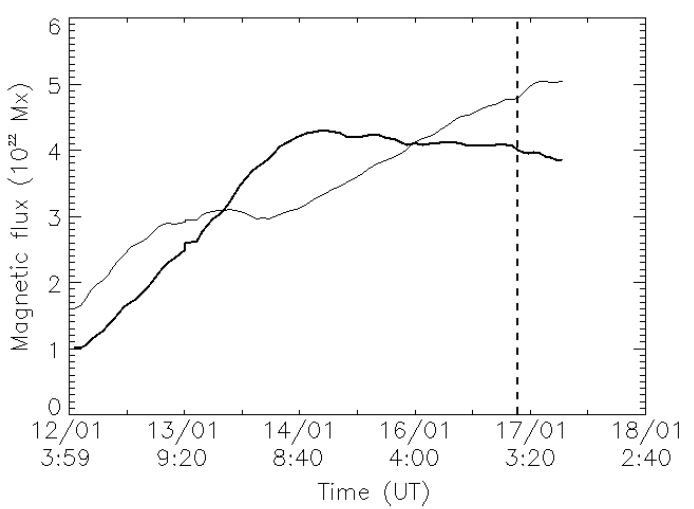

(a)

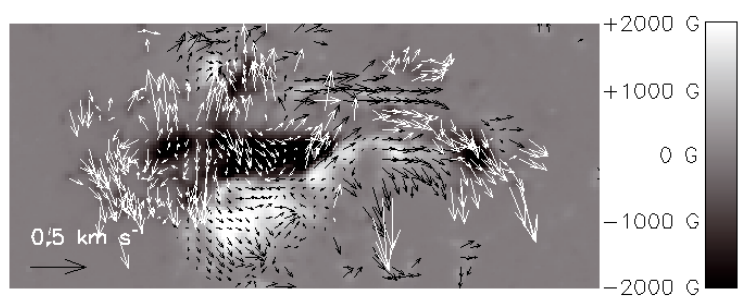

(b)

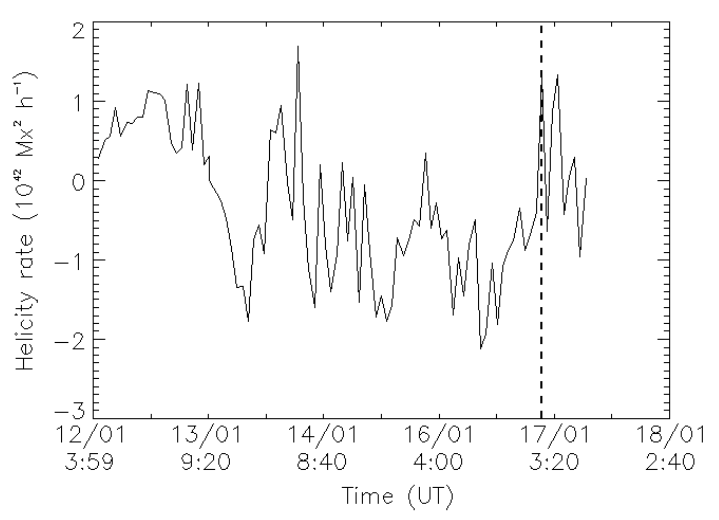

(c)

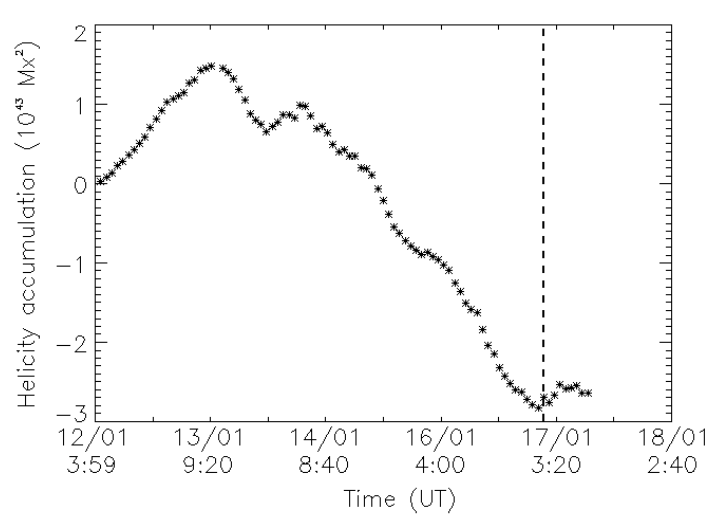

(d)

Fig. 7. a) Magnetic flux in NOAA 10720 as a function of time: the thin (thick) line indicates the negative (positive) flux. b) Horizontal velocity fields measured in NOAA 10720 on 17 January 2005; the fov is $400 \times 200$ arcsec. c) Magnetic helicity rate measured in NOAA 10720 during the period analyzed. d) Magnetic helicity accumulation in NOAA 10720. The vertical dotted lines indicate the time of occurrence of the X3.8 flare.
In this respect, we recall that Park et al. (2008) carried out a statistical study to investigate the variation in magnetic helicity over several days around the times of $11 \mathrm{X}$-class flares. They found that these flares were preceded by significant helicity accumulation $\left(1.8-16 \times 10^{42} \mathrm{Mx}^{2}\right)$ over a period spanning from 0.5 to a few days. Moreover, the helicity accumulated at a nearly constant rate $\left(4.5-48 \times 10^{40} \mathrm{Mx}^{2} \mathrm{~h}^{-1}\right)$, and successively assumed a nearly constant value before the flares.

One of the active regions analyzed by Park et al. (2008) was NOAA 10720: the authors demonstrated that since 16 January the active region was characterized by negative magnetic helicity injection, in agreement with our result (see also Wang et al. 2009). Moreover, Park et al. (2008) found that a phase of relatively constant helicity occurred before the event of 17th January. This last result however disagrees with the trend shown in Figs. 7c, $d$ and might be caused by the different techniques used to calculate the velocity field: in this paper, we used the DAVE technique, while Park et al. (2008) used the local correlation tracking (LCT) technique (November \& Simon 1988).

To investigate the configuration of the magnetic field of NOAA 10720 at coronal levels, we used an extrapolation code based on a method introduced by Alissandrakis (1981). The model assumes that the magnetic field is force-free both in the corona and at lower levels, and vanishes at infinity. Using the assumption of a constant $\alpha$ force-free field, the equation

$\nabla \times \boldsymbol{B}=\alpha \boldsymbol{B}$

can be numerically solved using Fourier transforms. In the code, the input parameters are the values of the longitudinal magnetic field component at the boundary (i.e. the photosphere), provided by MDI magnetograms. Therefore, it is possible to reconstruct the coronal magnetic field configuration, obtaining as output three $3 \mathrm{D}$ arrays with the values of each component of the magnetic field in 3D space.

Moreover, using a procedure that allows us to determine the localization of the null points in the magnetic configuration (Ugarte-Urra et al. 2007), we used the results obtained from the linear force-free field extrapolation and we could detect the presence of a low-lying null point in the western side of the active region (see Fig. 8).

More precisely, in Fig. 8 the colored lines show the location of a bundle of field lines connecting regions of different polarity in the central-western side of the active region. In Figs. 8a, b, these field lines are shown with different view angles and colors to highlight the different connectivity domains. In particular, Fig. $8 \mathrm{~b}$ clearly shows the location of a null point (at the intersection of the different color lines), which is located approximately above the region of contact between the filaments 1 and 2. Moreover, if we compare the location of the null point with the sites of brightness increase in $\mathrm{H}_{\alpha}$ (Fig. 4a) and X-ray (Fig. 4c) at 08:03 UT, we can deduce that these sites are spatially correlated with each other.

\section{Discussion}

To provide an overall description of the event under study, we highlight that from the GOES X-ray emission (0.5-4.0 $\AA$ ), we were able to infer that the event can be classified as a LDE, being characterized by a rise time of $\sim 3 \mathrm{~h}$ and a main phase duration of $\sim 3 \mathrm{~h}$. In particular, we interpret this event in the framework of recent models assuming that long duration events (LDEs) may result from continual magnetic reconnection driven by a positive feedback between a CME eruption and the fast magnetic reconnection inflow taking place beneath the erupting flux rope. 


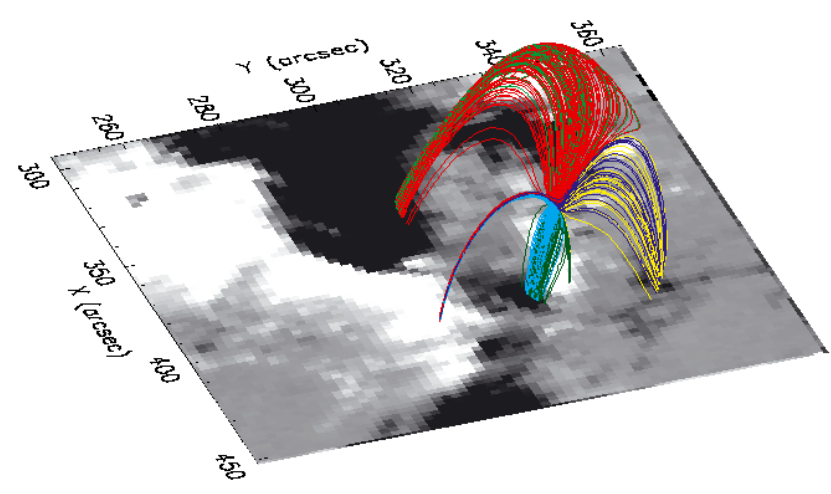

(a)

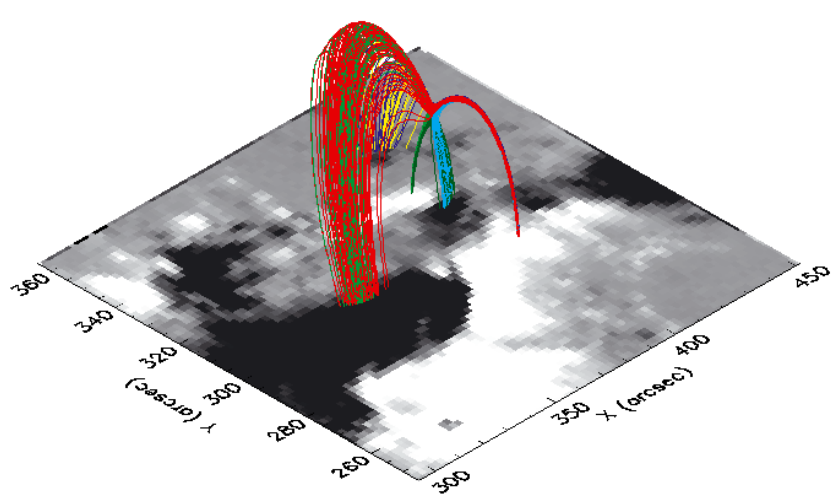

(b)

Fig. 8. Magnetic field lines and a null point overplotted on the MDI magnetogram acquired at 08:03 UT as seen from different view angles. The different colors indicate different connectivity domains. The null point is located at the intersection of the different color lines.

The analysis of $\mathrm{H}_{\alpha}$ and $1600 \AA$ images has uncovered ribbons that developed sequentially in different parts of the active region (in the central part at the very beginning, i.e. at 08:00 UT, in the eastern side after about one hour and in the western side after another $\sim 45 \mathrm{~min}$, see Fig. 9, third panel). From the $\mathrm{H}_{\alpha}$ images, we could also infer that the sites where the ribbons developed, previously hosted three filaments (named 1, 2, and 3 in Fig. 1c), and therefore we can interpret the ribbons as the footpoints of the arcades hosting these filaments. In this respect, we recall that the spatial resolution of the $\mathrm{H}_{\alpha}$ images did not allow us to distinguish whether the filaments were really separated, hence we cannot rule out the possibility that these features were portions of a single, highly sheared flux rope. However, in the following discussion, the physical processes that will be taken into account should have the same consequences in both cases (i.e., for different, but close flux ropes or for a single flux rope), owing to the common overall magnetic field configuration.

The $\mathrm{H}_{\alpha}$ images showed that the first filament was located in the region between sunspots $n_{3}$ and $p_{2}$ (see Fig. 1c), where hard X-ray emission during the rise phase was observed (see Fig. 5a). Moreover, from the linear force-free field extrapolations we could determine the presence of a null point whose position seems to overlap these features.

The photospheric footpoint motions reported in Fig. 7b indicate that in the region hosting filament 1 the velocity pattern was essentially parallel to the neutral line in the positive polarity, while it was diverging from the neutral line in the negative polarity. Previous studies of this active region evolution have shown that in the previous days the magnetic shear was also quite important, resulting in a complicated magnetic field configuration (Cheng et al. 2010b). Moreover, the graph shown in Fig. 7a indicates a continuous increase in the positive polarity in the previous days. From these results, it seems plausible to assume that the photospheric footpoint motions and new magnetic flux emergence might have caused the loss of stability of the arcade supporting this filament via tether-cutting reconnection of these field lines and caused its destabilization, that subsequently provoked reconnection at the null point and the rearrangement of the magnetic configuration of the active region.

Moreover, we recall that the flare analyzed in this paper was also studied by Liu et al. (2008), who analyzed the relationship between the flare intensity and the relevant active region complexity, which was measured by the parameter $\boldsymbol{E}=\boldsymbol{u} \times \boldsymbol{B}$, describing the dynamical evolution of both the velocity and magnetic fields. The authors found that, during the flare evolution, the flare kernels were localized near the magnetic inversion line, at sites where $\boldsymbol{E}$ was characterized by the highest values.

The analysis of the radio data showed dm type III radio bursts at about 8:41 UT, indicating the presence of electron beams at the plasma densities in the range $7.9 \times 10^{9}-4.9 \times$ $10^{10} \mathrm{~cm}^{-3}$. Successively, a series of reverse drift type III bursts were observed, indicating electron beams propagating from the corona towards denser chromosphere. Once these electron beams reached the chromosphere, they heated the plasma, such that the regions corresponding to the footpoints of the field lines increased their brightness and the $1600 \AA$ and $\mathrm{H} \alpha$ ribbons were observed.

As shown by radio observations, a series of so-called drifting pulsating structures (DPSs) were observed before the first CME (starting, on the basis of the back-extrapolated take-off time at 09:06 UT). Most of them had negative frequency drift. These radio features indicate that in the early phases of this CME an extended current sheet was formed and several plasmoids, moving upwards in the solar atmosphere, were generated inside this current sheet. These phenomena are indicative of fast reconnection and are related to the destabilization, rise, and eruption of filament 2 , with the consequent vertical current-sheet formation below the rising filament. During the early phases of the second CME (starting at 09:42 UT), there is no such observations. These radio structures were probably covered by the strong dmcontinuum observed at these times. However, at the time of the second CME, the analysis of the $\mathrm{H}_{\alpha}$ and HXR light curves, carried out at several ribbon locations, gave some indications of the fast electron beams and conduction fronts reaching the chromosphere, which might be associated with a reconnection process occurring at this stage.

We also recall the analysis carried out by Temmer et al. (2007), who studied the same event and focused their attention on the ribbons relevant to the destabilization and eruption of filament 3, and in particular to the time interval 09:43-10:02 UT, to determine the local reconnection and energy release rates. Temmer et al. (2007) found that the reconnection rate and the energy release rate were not uniform along these flare ribbons, but much larger at the locations where the hard X-ray footpoints were observed. The authors concluded that these results are consistent with a scenario where the electrons are accelerated primarily along some loop subsystems (outlined by the hard X-ray footpoints), while a minor fraction goes into the larger arcade whose footpoints are observed as diffuse bright ribbons in $\mathrm{H}_{\alpha}$ and $1600 \AA$ images. This result, if correlated with substantial dynamical evolution of the several bright $\mathrm{H}_{\alpha}$ and $1600 \AA$ 


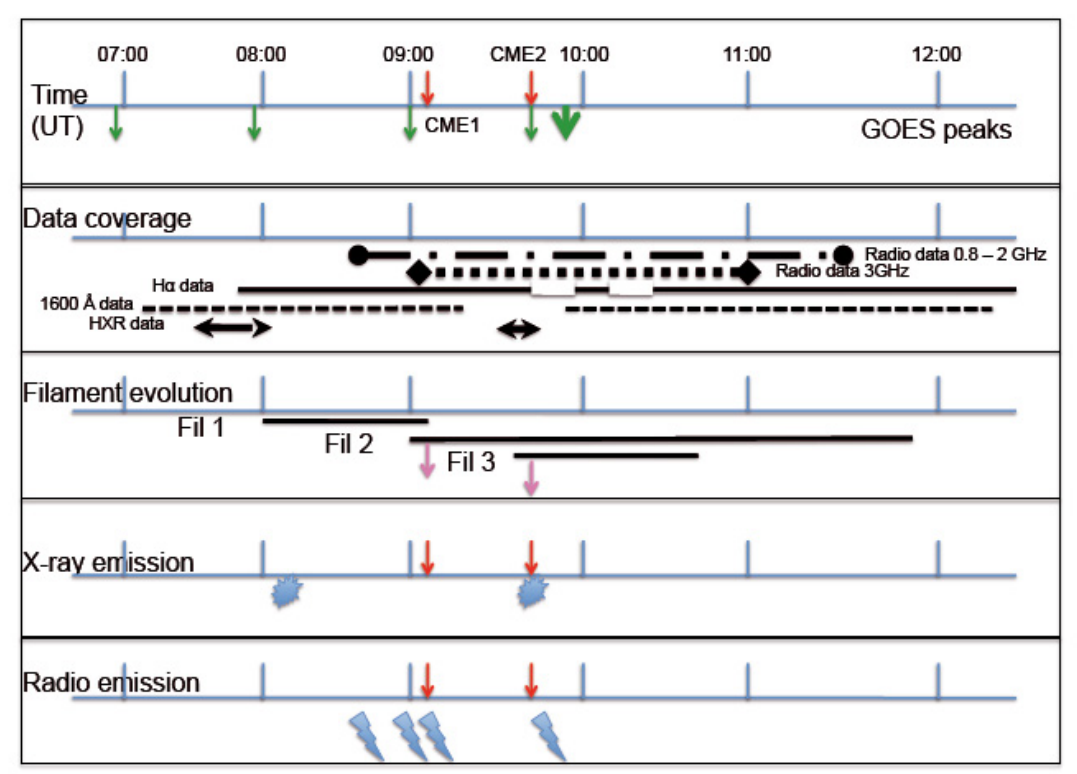

Fig. 9. Timeline of the the data coverage and of the main results deduced at different wavelengths. From top to bottom, first panel: timeline indicating the GOES X-ray peaks (green arrows; the greater green arrow indicates the time of the peak of the X3.8 flare, while the red arrows indicate the back-extrapolated take-off times of the CMEs). Second panel: data coverage during the LDE (the dotted-continuous line indicates radio data acquired in the range $0.8-2.0 \mathrm{GHz}$; the dotted line indicates radio data at $3 \mathrm{GHz}$; the continuous line indicates the $\mathrm{H}_{\alpha}$ data coverage (there are two gaps: 09:50-09:58 UT and 10:07-10:25 UT); the broken line indicates the $1600 \AA$ TRACE data coverage; the lines with arrows indicate the RHESSI HXR coverage). Third panel: time intervals relevant to the three filament destabilization or eruption; the magenta arrows indicate the times of the eruptions of filaments 2 and 3. Fourth and fifth panels indicate with symbols the main emission in the HXR and radio ranges, respectively (see text).

ribbons observed during the entire event, and to the limited hard $\mathrm{X}$-ray sources observed, might be indicative of a scenario where the destabilization process and the consequent reconnections occurred sequentially at different locations during the LDE (compare with Fig. 5).

To summarize this scenario, the slow reconnection due to the tether-cutting of the field lines below the central part of the flux rope and the rearrangement of the field lines at the null point, caused a change in the connectivity of the magnetic field lines in the active region, hence a destabilization of the arcades that supported the other two filaments, firstly the filament 2, located in the eastern side of the active region, and later the filament 3 , located in the western side, in a kind of domino effect (see, e.g.; Liu et al. 2009, Paper I). The overall restructuring of the magnetic field of the active region and the destabilizations and eruptions of filaments 2 and 3 caused the two CMEs, which were ejected at 09:06 UT and 09:42 UT, respectively.

\section{Conclusions}

Several models of CMEs assume that there is a flux rope with a magnetic configuration characterized by a Lorentz self-force; this flux rope has a tendency to erupt, but this force is balanced by the overlying background magnetic field, which provides the downward Lorentz force. When the background magnetic field gradient (also called the decay index) reaches a certain threshold, a torus or partial torus instability may set-in (Cheng et al. 2011).

Cheng et al. (2011) studied the nine M and X-class flares that occurred in NOAA 10720, six of which were confined and three of which were eruptive. These authors concluded that the confined flares occurred closer to the active region center, while the eruptive flares took place at its periphery. Moreover, Cheng et al. (2011) demonstrated that, at the same height in the corona, the decay index is higher in the centre of the active region and lower at its outskirts, while the strength of the transverse overlying magnetic field is higher in the central part of the active region, and it decreases at its periphery. As a consequence, it is more difficult for a flux rope to erupt when it is situated at the center of the active region, because of the stronger overlying transverse magnetic field and the smaller decay index. In contrast, when the flux rope is located at the periphery of the active region, its eruption is easier because of the weaker overlying transverse magnetic field and the higher decay index. A particular case of this scenario is when only portions of the flux rope, on the outskirts of the active region, can erupt.

We note that the change in the magnetic helicity flux observed after the flare, was probably due to the restoring of the torque balance between the coronal field and the subphotospheric portion of the flux tubes, once the two CMEs were ejected (see Smyrli et al. 2010).

Taking into account the sequence of phenomena observed at various wavelengths in the event under study, we propose that because of its complexity, its long duration, the sequence of filaments destabilization, and the ejection of two CMEs, it can be interpreted in the framework of the following scenario:

- Magnetic flux emergence and photospheric shearing motions occurring mainly in the surroundings of the inversion line where the (central) flux rope is lying, combine to form slow phase of magnetic energy storage and might eventually cause a slow tether-cutting phase with consequent magnetic reconnection and re-arrangement of the magnetic configuration.

- A tether-cutting process below the central flux rope begins in the central part of the active region, resulting in several signatures observed at different wavelengths $\left(\mathrm{H}_{\alpha}\right.$ and $1600 \AA$ ribbons, HXR emission). 
- The flux rope, however, is unable to erupt, despite the slow magnetic reconnection caused by the tether-cutting process that has been taking place in this area for several hours. The strength of the transverse, overlying magnetic field, in the area is the highest in the active region.

- The presence in this area of a null point, inferred from force-free field extrapolations, indicating that here the magnetic field configuration has the highest degree of complexity, might have a role in the re-arrangement of the magnetic field line connectivity, transferring the destabilization process to the other bundles of field lines sustaining the other filaments/flux ropes.

- The destabilization of the (central) flux rope reaches the periphery of the active region and here, at both its eastern and western sides, where, respectively, filaments 2 and 3 were observed, the conditions, in terms of the strength of the overlying transverse magnetic field and the decay index, are such that they are destabilized and later erupted, giving rise to the two observed CMEs.

This LDE can therefore be considered as an example of a domino process characterized by destabilizations in successive steps, giving rise to consecutive episodes of energy release. It would be interesting to investigate whether a similar behavior can be found in other active regions where several filaments are present and investigate whether the number of filaments, their extension and their possible fragmentation might provide reliable predictions of very energetic, long-duration events. Finally, we stress the importance of these studies in the framework of space weather phenomena that might have severe impacts on the Earth environment, especially in the coming years as we approach a new solar maximum and there will be an increasing probability of the formation of complex active regions where these phenomena are more likely to occur. In order to reach the previously mentioned goals, both high-resolution satellite data, such as those provided by RHESSI, HINODE, and SDO, as well as those that could be provided by ground-based instruments are necessary, until the new generation of 4-m telescopes, such as EST and ATST, become available.

Acknowledgements. This work was supported by the European Commission through the SOLAIRE Network (MRTN-CT-2006-035484), by the Istituto Nazionale di Astrofisica (INAF), by the Catania University, by the Agenzia Spaziale Italiana (contract I/035/05/0) and by Grant IAA300030701 of the Grant Agency of the Academy of Sciences of the Czech Republic. Thanks are also due to E. Catinoto and P. Costa for their cooperation in acquiring photospheric and $\mathrm{H} \alpha$ images at the INAF-Catania Astrophysical Observatory.

\section{References}

Alissandrakis, C. E. 1981, A\&A, 100, 197

Aschwanden, M. J. 2004, Physics of the Solar Corona (Springer)

Bárta, M., \& Karlický, M. 2001, A\&A, 379, 1045
Brueckner, G. E., Howard, R. A., Koomen, M. J., et al. 1995, Sol. Phys. 162, 357 Carmichael, H. 1964, in Proc. of the AAS-NASA Symp. - The Physics of Solar Flares, ed. W. N. Hess, Washington, National Aeronautics and Space Administration, Science and Technical Information Division, 451

Chae, J. 2001, ApJ, 560, L95

Chae, J., Moon, Y. J., \& Park, Y. D. 2004, Sol. Phys., 223, 39

Cheng, X., Ding, M. D., \& Zhang, J. 2010a, ApJ, 712, 1302

Cheng, X., Ding, M. D., Guo, Y., et al. 2010b, ApJ, 716, L68

Cheng, X., Zhang, J., Ding, M. D., Guo, \& Y., Su, J. T. 2011, ApJ, in press

Criscuoli, S., Romano, P., Giorgi, F., \& Zuccarello, F. 2009, A\&A, 506, 1429

Delaboudinière, J., Artzner, G. E., Brunaud, J., et al., 1995, Sol. Phys., 162, 291

Domingo, V., Fleck, B., \& Poland, A. I., 1995, Sol. Phys., 162, 1

Freeland, S. L., \& Handy, B. N. 1998, Sol. Phys., 182, 497

Handy, B. N., Acton, L. W., Kankelborg, C. C., et al. 1999a, Sol. Phys., 187, 229

Handy, B. N., Tarbell, T. D., Wolfson, C. J., Korendyke, C. M., \& Vourlidas, A. 1999b, Sol. Phys., 190, 351

Heyvaerts, J., Priest, E. R., \& Rust, D. M. 1977, ApJ, 216, 123

Hirayama, T. 1974, Sol. Phys., 34, 323

Hirose, S., Uchida, Y., Uemura, S., Yamaguchi, T., \& Cable, S. B. 2001, ApJ, 551586

Hurford, G. J., Schmahl, E. J., Schwartz, R. A., et al. 2002, Sol. Phys., 210, 61

Jiřička, K., Karlický, M., Kepka, O., \& Tlamicha, A. 1993, Sol, Phys., 147, 203

Karlický, M. 1984, Sol. Phys., 92, 329

Karlický, M. 2004, A\&A, 417, 325

Karlický, M., Sobotka, M., \& Jiřička, K. 1996, Sol. Phys., 168, 375

Karlický, M., Bárta, M., Jiřička, K., et al. 2001, A\&A, 375, 638

Kitahara, T., \& Kurokawa, H. 1999, Sol. Phys., 125, 321

Kliem, B., Karlický, M., \& Benz, A. O. 2000, A\&A, 360, 715

Kopp, R. A., \& Pneuman, G. W. 1976, Sol. Phys., 50, 85

Lin, J. 2004, Sol. Phys., 219, 169

Lin, R. P., Dennis, B. R, Hurford, G. Y., et al. 2002, Sol. Phys., 210, 3

Liu, Ch., Lee, J., Karlický, M., et al. 2009, ApJ., 703, 757

Liu, J., Zhang, Y., \& Zhang, H., 2008, Sol. Phys., 248, 67

Lopez Fuentes, M. C., Demoulin, P., Mandrini, C. H., \& van Driel-Gesztelyi, L. 2000, ApJ, 544, 540

Maričić, D., Vršnak, B., Stanger, A. L., et al. 2007, Sol. Phys., 241, 99

November, L. J., \& Simon, G. W. 1988, ApJ, 333, 427

Park, S.-H., Lee, J., Choe, G. S., et al. 2008, ApJ., 686, 1397

Priest, E. R., \& Forbes, T. G. 2000, Magnetic Reconnection: MHD Theory and Applications (Cambridge University Press)

Priest, E. R., \& Forbes, T. G. 2002, A\&AR, 10, 313

Priest, E. R., Parnell, C. E., \& Martin, S. F. 1994, ApJ., 427, 459

Rausche, G., Aurass, H., Mann, G., Karlický, M., \& Vocks, C. 2007, Sol. Phys., 245,327

Romano, P., \& Zuccarello, F. 2007, A\&A, 474, 633

Scherrer, P. H., Bogart, R. S., Bush, R. I., et al. 1995, Sol. Phys., 162, 129

Schuck, P. W. 2005, ApJ, 632, L53

Smyrli, A., Zuccarello, F., Romano, P., et al. 2010, A\&A, 521, A56

Sturrock, P. A. 1966, Nature, 211, 695

Temmer, M., Veronig, A. M., Vršnak, B., \& Miklenic, C. 2007, ApJ, 654, 665

Temmer, M., Veronig, A. M., Vršnak, B., et al. 2008, ApJ, 673, L95

Temmer, M., Vršnak, B., Žic, T., \& Veronig, A. M. 2009, ApJ, 702, 1343

Török, T., Berger, M. A., \& Kliem, B. 2010, A\&A, 516, A49

Ugarte-Urra, I., Warren, H. P., \& Winebarger, A. R. 2007, ApJ, 662, 1293

Veronig, A. M., Temmer, M., Vršnak, B., \& Thalmann, J. K. 2006, ApJ, 647, 1466

Vršnak, B. 1990, Sol. Phys., 129, 195

Vršnak, B., Maričić, D., Stanger, A. L., \& Veronig, A. M. 2004, Sol. Phys., 225, 355

Wang, J., Zhao, M., \& Zhou, G. 2009, ApJ., 690, 862

Zhao, M., Wang, J.-X., Matthews, S., et al. 2009, Res. Astron. Astrophys., 9, 812

Zuccarello, F., Romano, P., Farnik, F., et al. 2009, A\&A, 493, 629 (Paper I) 